\title{
Multifunctional/Multitargeted Anticancer Agent OMN54
}

National Cancer Institute

\section{Source}

National Cancer Institute. Multifunctional/Multitargeted Anticancer Agent OMN54. NCI

Thesaurus. Code C101260.

An orally available, multivalent herbal formulation containing a novel mixture of whole extracts from three commonly used Chinese medicinal herbs Ganoderma lucidum (lingzhi mushroom), Salvia miltiorrhiza (Chinese sage, or danshen) and Scutellaria barbata (ban zhi lian), with potential immunomodulating, antiangiogenic, anti-inflammatory, antiproliferative and antiviral activities. Although the exact mechanism of action remains to be fully elucidated due to the complexity of the multiple phytochemicals, multifunctional/multitargeted anticancer agent OMN54 appears to work in an additive and synerg istic manner by acting on a variety of signaling pathways and on multiple targets, such as vascular endothelial growth factor, nuclear factor kappa B, interleukin1 beta, fibroblast growth factor, and epidermal growth factor. 